TITLE:

\title{
Limiting Results for the Free Energy of Directed Polymers in Random Environment with Unbounded Jumps
}

\section{$\operatorname{AUTHOR}(S)$ :}

Comets, Francis; Fukushima, Ryoki; Nakajima, Shuta; Yoshida, Nobuo

\section{CITATION:}

Comets, Francis ...[et al]. Limiting Results for the Free Energy of Directed Polymers in Random Environment with Unbounded Jumps. Journal of Statistical Physics 2015, 161(3): 577-597

\section{ISSUE DATE:}

2015-11

URL:

http://hdl.handle.net/2433/241738

\section{RIGHT:}

This is a post-peer-review, pre-copyedit version of an article published in 'Journal of Statistical Physics'. The final authenticated version is available online at: https://doi.org/10.1007/s10955-015-1347-1.; The full-text file will be made open to the public on 09 August 2016 in accordance with publisher's 'Terms and Conditions for Self-Archiving'.; This is not the published version. Please cite only the published version.; この論文は出版社版でありません。引用の際には出 版社版をご確認ざ利用ください。 


\title{
LIMITING RESULTS FOR THE FREE ENERGY OF DIRECTED POLYMERS IN RANDOM ENVIRONMENT WITH UNBOUNDED JUMPS
}

\author{
FRANCIS COMETS, RYOKI FUKUSHIMA, SHUTA NAKAJIMA, AND NOBUO YOSHIDA
}

\begin{abstract}
We study asymptotics of the free energy for the directed polymer in random environment. The polymer is allowed to make unbounded jumps and the environment is given by Bernoulli variables. We first establish the existence and continuity of the free energy including the negative infinity value of the coupling constant $\beta$. Our proof of existence at $\beta=-\infty$ differs from existing ones in that it avoids the direct use of subadditivity. Secondly, we identify the asymptotics of the free energy at $\beta=-\infty$ in the limit of the success probability of the Bernoulli variables tending to one. It is described by using the so-called time constant of a certain directed first passage percolation. Our proof relies on a certain continuity property of the time constant, which is of independent interest.
\end{abstract}

\section{INTRODUCTION AND MAIN RESULTS}

The directed polymer in random environment is a statistical physics model of a polymer in disordered solvent. In the discrete set-up, the polymer chain is a random walk $\left(\left(X_{n}\right)_{n \geq 0}, P\right)$ on $\mathbb{Z}^{d}$ starting at the origin and the random environment is modelled by independent and identically distributed random variables $\left((\eta(j, x))_{(j, x) \in \mathbb{N} \times \mathbb{Z}^{d}}, Q\right)$. We introduce the Hamiltonian $H_{n}^{\eta}=\sum_{j=1}^{n} \eta\left(j, X_{j}\right)$ and, for a given inverse temperature $\beta \in \mathbb{R}$, define the finite volume Gibbs measure by

$$
\mathrm{d} \mu_{n}^{\eta, \beta}=\frac{1}{Z_{n}^{\eta, \beta}} \exp \left\{\beta H_{n}^{\eta}\right\} \mathrm{d} P,
$$

where $Z_{n}^{\eta, \beta}=P\left[\exp \left\{\beta H_{n}^{\eta}\right\}\right]$ is the partition function with $P[\cdot]$ denoting the expectation with respect to $P$. When $\beta>0$, the polymer is attracted by large values of $\eta$ and repelled by negative values. It is known that this interaction causes a localization transition depending on the law of the random walk [10].

A quantity of particular importance in this model is the free energy

$$
\varphi(\beta)=\lim _{n \rightarrow \infty} \frac{1}{n} \log Z_{n}^{\eta, \beta}
$$

Date: August 11, 2015.

2010 Mathematics Subject Classification. Primary 60K37; secondary 60K35; 82A51; 82D30.

Key words and phrases. directed polymer, random environment, first passage percolation, ground states, zero temperature. 
whose existence is usually established by a subadditivity argument. It is for instance believed that the difference between $\varphi(\beta)$ and the so-called annealed free energy characterizes the localized/delocalized phases. See $[3,8,6,11]$ for rigorous results in this direction.

1.1. Zero temperature limits and open paths counting. One of the main results in the present article is about the zero temperature limit of the free energy $\varphi(\beta)$. Let us give a few words on the motivation. There has recently been a revival of interest in the problem concerning the number of extremal paths in random media that dates back to [20, 17], see for example [7, 32, 25, 26, 18, 19] for recent works in the directed setup. Among others, Garet-Gouéré-Marchand [19] have recently established the existence of the growth rate of the number of open paths in nearest neighbor oriented percolation. To be more precise, let $N_{n}$ be a number of open paths of length $n$ starting from $(0,0) \in \mathbb{N} \times \mathbb{Z}^{d}$. Then assuming that the percolation takes place with a positive probability, it is proved that $\lim _{n \rightarrow \infty} n^{-1} \log N_{n}$ exists and is non-random on the event of percolation. The main difficulty is that the standard subadditivity argument does not work as $\log N_{n}$ is not well-defined (or should be defined as $-\infty$ ) with positive probability, making this quantity not integrable. One of the motivations of the present work is to propose an approach to the same problem by considering the zero temperature limit of the directed polymer model. Indeed, when the random walk is simple nearest neighbor walk and $\eta$ is a Bernoulli variable, the above partition function at $\beta=-\infty$ coincides with $(2 d)^{-n} N_{n}$. If we are able to prove that the convergence

$$
\frac{1}{n} \log Z_{n}^{\eta, \beta} \rightarrow \frac{1}{n} \log N_{n}-\log 2 d \text { as } \beta \rightarrow-\infty
$$

is uniform in $n$ on the event of percolation, then it follows that $\lim _{n \rightarrow \infty} n^{-1} \log N_{n}$ exists and is equal to $\lim _{\beta \rightarrow-\infty} \varphi(\beta)+\log 2 d$. In this paper, we carry out this program for random walks with stretched-exponential transition probabilities as a test case. The unboundedness of jumps simplifies the problem since no percolation transition occurs anymore. However we note that our approach automatically yields the stronger continuity result of the free energy at $\beta=-\infty$. One of the reasons for our rather special choice of the transition probability is that with this choice, the model has a relation to a directed version of the first passage percolation studied in $[21,22]$, which is interesting in its own right. See Theorem 2 below.

We shall comment more on related works in Subsection 1.3 after describing our setting and results.

1.2. Setting and Results. Let $\left(\left\{X_{n}\right\}_{n \in \mathbb{N}}, P_{x}\right)$ be the random walk on $\mathbb{Z}^{d}$ starting from $x$ and with the transition probability

$$
P_{x}\left(X_{n+1}=z \mid X_{n}=y\right)=f\left(|y-z|_{1}\right),
$$

where $f: \mathbb{N} \cup\{0\} \rightarrow(0,1)$ is a function of the form

$$
f(k)=c_{1} \exp \left\{-c_{2} k^{\alpha}\right\}, \quad \text { where } \alpha>0 .
$$


We write $P$ instead of $P_{0}$ for simplicity.

Remark 1. Our choice of the jump law is somewhat arbitrary, and it is tempting to replace our specific choice with some regular variation assumption on the tail of $\log f(k)$. It is a purely technical exercise to adapt our method in order to cover such cases. To make arguments as transparent as possible we stick to this simple law.

In view of the motivation explained above, we assume that $\left(\{\eta(j, x)\}_{(j, x) \in \mathbb{N} \times \mathbb{Z}^{d}}, Q\right)$ is independent and identically distributed Bernoulli random variables with

$$
Q(\eta(0,0)=1)=p \in(0,1) .
$$

We define the partition functions at $\beta=-\infty$ by

$$
Z_{n}^{\eta,-\infty}=P\left(H_{n}^{\eta}=0\right)
$$

in addition to the notation introduced before. Note that $Z_{n}^{\eta,-\infty}$ is positive for $Q$ almost every $\eta$, since the random walk has unbounded jumps. It is routine to show that, $Q$-almost surely and for all $\beta \in \mathbb{R}$, the free energy exists and is equal to the second line:

$$
\begin{aligned}
\varphi(p, \beta) & =\lim _{n \rightarrow \infty} \frac{1}{n} \log Z_{n}^{\eta, \beta} \\
& =\lim _{n \rightarrow \infty} \frac{1}{n} Q\left[\log Z_{n}^{\eta, \beta}\right] .
\end{aligned}
$$

Then, it is plain to see that $\varphi$ is non-decreasing in $p$ for $\beta>0$, non-increasing in $p$ for $\beta<0$, non-decreasing and convex in $\beta$, and that $\varphi(p, \beta)=\varphi(1-p,-\beta)+\beta$ for $\beta$ real. Furthermore, one can show by a simple application of the so-called block argument that

$$
\lim _{\beta \rightarrow-\infty} \varphi(p, \beta) \geq \liminf _{n \rightarrow \infty} \frac{1}{n} \log Z_{n}^{\eta,-\infty}>-\infty .
$$

See Appendix for a proof. Our first result shows that the free energy exists and is jointly continuous in $(p, \beta)$, including $\beta=-\infty$.

Theorem 1. In the above setting with $\alpha \in(0, d)$, the limit

$$
\varphi(p,-\infty)=\lim _{n \rightarrow \infty} \frac{1}{n} \log Z_{n}^{\eta,-\infty}
$$

exists $Q$-almost surely. Moreover, the function $\varphi(p, \beta)$ is jointly continuous on $(0,1] \times[-\infty, \infty) \backslash\{(1,-\infty)\}$.

It is possible to show the first part for general $\alpha \in(0, \infty)$ by using the subadditive ergodic theorem, as in the proof of Theorem 2.1 of [15], with the help of the fact that

$$
Q\left[\left|\log Z_{n}^{\eta,-\infty}\right|\right]<\infty .
$$

However, we prove it as a part of the proof of continuity result, avoiding direct use of the subadditive ergodic theorem at $\beta=-\infty$. As explained above, we think this is of technical importance. Note that the above integrability condition may break 
down even for a model where there is no percolation transition. The Brownian directed polymer in Poissonian medium with $\beta=-\infty$ is such an example, as one can easily check by considering the event that there is a Poissonian trap very close to the origin.

Note that at the exceptional point in Theorem $1, \varphi$ should be defined as $\varphi(1,-\infty)=$ $-\infty$. It is then natural to ask how $\varphi(p, \beta)$ grows as $(p, \beta) \rightarrow(1,-\infty)$. Our next result addresses a directional asymptotics. Note that $\varphi(\beta, p)$ exists $Q$-a.s. for all $\alpha>0$, as we have just mentioned.

Theorem 2. In the above setting with $\alpha \in(0, \infty)$, there exists a constant $\mu_{1}>0$ such that as $p \uparrow 1$,

$$
\varphi(p,-\infty) \sim-c_{2} \mu_{1}(1-p)^{-\alpha / d} .
$$

The constant $c_{2}$ comes from (1.2), and $\mu_{1}$ is defined by (1.7) with $p=1$.

Remark 2. If we replace $\eta$ by $1-\eta$ and denote the corresponding free energy by $\tilde{\varphi}(p, \beta)$, we can deduce its asymptotics as $\beta \rightarrow+\infty$ and $p \downarrow 0$ from Theorem 1 and 2 as follows:

$$
\lim _{\beta \rightarrow+\infty}(\tilde{\varphi}(p, \beta)-\beta) \text { exists and asymptotic to }-c_{2} \mu_{1} p^{-\alpha / d} \text { as } p \downarrow 0 .
$$

This kind of symptotics are extensively studied in the continuous time setting, see Subsection 1.3 below. In the discrete time setting, however, this is the first result in the same direction to the best of our knowledge - possibly because for the common nearest neighbor walk model, the high density asymptotics at $\beta=-\infty$ is trivial. Moreover, we encounter a new directed first passage percolation model in identifying the constant $\mu_{1}$ which is interesting in its own right. Let us explain how it comes into play.

The asymptotics (1.5) has a simple heuristic interpretation. When $p$ is close to 1 , the sites at which $\eta=0$ have low density $1-p$ and hence the random walk has to make a jump of order $(1-p)^{-1 / d}$ at each step to achieve $H_{n}^{\eta}=0$. The probability of such a path decays like $\exp \left\{-(1-p)^{-\alpha / d} n\right\}$ and this explains the $p$-dependent factor. In fact, it turns out that the main contribution to the free energy comes from the path which carries the highest probability and hence the constant $c_{2} \mu_{1}$ corresponds to the growth rate of the minimal cost for the random walk.

Note that this minimal cost could in principle depend on $p$, but actually it does not, as we will see in the next theorem. There, we prove the continuity as $p \uparrow 1$ of the time constant of a certain directed first passage percolation, a result of independent interest. Denote the (scaled) points where the random walk is allowed to go by

$$
\omega_{p}=\sum_{(k, x) \in \mathbb{N} \times \mathbb{Z}^{d}}(1-\eta(k, x)) \delta_{\left(k, s_{p} x\right)},
$$

with the natural scaling factor $s_{p}=\left(\log \frac{1}{p}\right)^{1 / d} \sim(1-p)^{1 / d}(p \uparrow 1)$. With some abuse of notation we will frequently identify $\omega_{p}$, and more generally any point measure, 
with its support. Given a realization of $\omega_{p}$, we define the passage time from 0 to $n$ by

$$
T_{n}\left(\omega_{p}\right)=\min \left\{\sum_{k=1}^{n}\left|x_{k-1}-x_{k}\right|^{\alpha}: x_{0}=0 \text { and }\left\{\left(k, x_{k}\right)\right\}_{k=1}^{n} \subset \omega_{p}\right\} .
$$

Then, a direct application of the subadditive ergodic theorem shows that the limit

$$
\mu_{p}=\lim _{n \rightarrow \infty} \frac{1}{n} T_{n}\left(\omega_{p}\right)
$$

exists $Q$-almost surely. The limit $\mu_{p}$, so-called time constant, is deterministic. In these terms, the maximal probability of paths satisfying $H_{n}^{\eta}=0$ is expressed as

$$
c_{1}^{n} \exp \left\{-c_{2} s_{p}^{-\alpha} T_{n}\left(\omega_{p}\right)\right\}=\exp \left\{-c_{2} \mu_{p}(1-p)^{-\alpha / d} n(1+o(1))\right\} .
$$

Now note that $\omega_{p}$ converges as $p \uparrow 1$ to the Poisson point process $\omega_{1}$ on $\mathbb{N} \times \mathbb{R}^{d}$ whose intensity is the product of the counting measure and Lebesgue measure. Observe also that definition (1.6) makes perfect sense when $p=1$, yielding a limit $\mu_{1}$ in (1.7). In the next result we claim that the time constant of the Bernoulli model converges to that of the Poisson model as $p \uparrow 1$.

Theorem 3 (Continuity of the time constant). We have

$$
\lim _{p \uparrow 1} \mu_{p}=\mu_{1} .
$$

Remark 3. A similar continuity of the time constant is known for lattice first passage percolation in greater generality, see $[12,13]$ and $(6.9)$ in $[24]$.

1.3. Related works. The main part of Theorem 1 is the continuity of $\varphi(p, \beta)$ around $\beta=-\infty$, which is the zero temperature asymptotic result for the free energy. This type of problems does not seem to attract much interest in the discrete time setting since in some cases the answers are simple. For instance, consider the (nearest-neighbor) simple random walk model with an i.i.d. random environment with $Q(\eta(0,0)>0)>0$. Then it is easy to see that as $\beta \rightarrow+\infty$, the free energy is asymptotic to $\beta$ times the time constant of the directed last passage percolation. However, if $\eta$ is Bernoulli distributed and we send $\beta \rightarrow-\infty$, the situation is not so simple. As we mentioned at the beginning, the existence of $\varphi(-\infty)$ proved in [19] is already highly nontrivial and the continuity as $\beta \rightarrow-\infty$ remains an open question at the moment.

For the continuous time polymer models, the asymptotics of the free energy is far from being simple. Continuous time random walk models, known under the name of parabolic Anderson model, have attracted enormous attention. CarmonaMolchanov in the seminal work [5] initiated this line of research. They mainly studied the case when the environment is a space-time Gaussian white noise and their results include non-matching upper and lower bounds for the free energy when the jump rate of the random walk tends to zero. Note that this limit is similar to that in Theorem 2 in spirit since in both cases, the random walk is forced to make 
more jumps than it typically does. Shiga [31] proved similar results for the spacetime Poissonian environment at $\beta=-\infty$. In fact, both [5] and [31] only proved the existence of the free energy in the sense of a $L^{1}$ limit. These results were later refined and extended in $[28,15,16,14]$ and almost sure existence of the free energy was established in $[15,16]$. Finally, the sharp equivalent for the free energy as the jump rate vanishes was obtained in $[4,15]$ in terms of the time constant of a last passage percolation problem. Note that for the Gaussian white noise environment, the above asymptotics is readily translated to the $\beta \rightarrow \pm \infty$ limit by using a scaling identity (see Chapter IV of [5]). On the other hand, in the Poissonian environment case, these zero temperature limits are of independent interest but have not been considered yet. In particular, we expect that the continuity similar to Theorem 1 holds when $\beta \rightarrow-\infty$.

Another continuous time polymer model is Brownian directed polymer in Poissonian environment introduced by Comets-Yoshida [9]. The $\beta \rightarrow+\infty$ limit was studied in the same paper, as well as $\beta \rightarrow-\infty$ for $d \geq 3$ with a specific choice of the other parameters. It is possible to show by a block argument that the finite volume free energy stays bounded as $\beta \rightarrow-\infty$ in general but, to the best of our knowledge, the existence of the limit at $\beta=-\infty$ is not known. Later in [11], the asymptotics as the density of the Poisson point process tends to $\infty$ was also studied but only for bounded $\beta$, in contrast to Theorem 2 here.

Finally, we mention that some solvable models have been found recently, see, e.g., Moriarty-O'Connell [27], Amir-Corwin-Quastel [1] and Seppäläinen [30]. In these models the free energy can be explicitly computed, thus allowing to study various asymptotics. But we refrain from explaining the details of these results since such examples have been found only in $(1+1)$-dimension so far and also the techniques employed are quite different from ours.

1.4. Organization of the paper. The rest of the paper is organized as follows. Section 2 is devoted to the proof of Theorem 1 . For $\beta \in \mathbb{R}$, the continuity is relatively easy and the essential part is the proof of continuity around $\beta=-\infty$. The basic strategy is to introduce a deformation of the path with a quantitative control of the resulting error. In Section 3, we prove Theorem 3, as well as a concentration result which is used in the proof of Theorem 2. Finally, we prove Theorem 2 in Section 4, by showing that the heuristic computation given below Remark 1.4 is indeed correct. There, we closely follow arguments of Mountford [28].

\section{Proof of Theorem 1}

Theorem 1. Note first that continuity in $\beta \in(-\infty, \infty)$ follows from convexity of $\varphi(p, \cdot)$. Next, we verify the continuity in $p$, locally uniformly in $\beta$, cf. (2.1) below. For this purpose, we take arbitrary $0<p<q \leq 1$, and introduce another family of independent and identically distributed Bernoulli variables $\left(\{\zeta(j, x)\}_{(j, x) \in \mathbb{N} \times \mathbb{Z}^{d}}, Q^{\prime}\right)$ with $Q^{\prime}(\zeta(0,0)=1)=(q-p) /(1-p)$ and define $\check{\eta}=\eta \vee \zeta$. Then, $\left(\{\check{\eta}(j, x)\}_{(j, x) \in \mathbb{N} \times \mathbb{Z}^{d}}, Q \otimes\right.$ 
$\left.Q^{\prime}\right)$ is a collection of Bernoulli random variables with success probability $q$ and we are going to estimate

$$
Q \otimes Q^{\prime}\left[\log Z_{n}^{\check{\eta}, \beta}-\log Z_{n}^{\eta, \beta}\right]=Q \otimes Q^{\prime}\left[\log \mu_{n}^{\eta, \beta}\left[\exp \left\{\beta H_{n}^{\check{\eta}-\eta}\right\}\right]\right],
$$

where $\mathrm{d} \mu_{n}^{\eta, \beta}=\left(Z_{n}^{\eta, \beta}\right)^{-1} \exp \left\{\beta H_{n}^{\eta}\right\} \mathrm{d} P$ is the polymer measure. For positive $\beta$, we have by Jensen's inequality that

$$
\begin{aligned}
0 & \leq Q \otimes Q^{\prime}\left[\log \mu_{n}^{\eta, \beta}\left[\exp \left\{\beta H_{n}^{\check{\eta}-\eta}\right\}\right]\right] \\
& \leq \log Q \otimes Q^{\prime}\left[\mu_{n}^{\eta, \beta}\left[\exp \left\{\beta H_{n}^{\check{\eta}-\eta}\right\}\right]\right] \\
& \leq \log Q \otimes Q^{\prime}\left[\mu_{n}^{\eta, \beta}\left[\exp \left\{\beta H_{n}^{\zeta}\right\}\right]\right] \\
& =\log Q\left[\mu_{n}^{\eta, \beta}\left[Q^{\prime}\left[\exp \left\{\beta H_{n}^{\zeta}\right\}\right]\right]\right] \\
& =n \log \left[\left(e^{\beta}-1\right)(q-p)+1\right] .
\end{aligned}
$$

For negative $\beta$, we again use Jensen's inequality for fixed $\eta$ and $\zeta$ to get

$$
\begin{aligned}
0 & \geq Q \otimes Q^{\prime}\left[\log \mu_{n}^{\eta, \beta}\left[\exp \left\{\beta H_{n}^{\check{\eta}-\eta}\right\}\right]\right] \\
& \geq Q \otimes Q^{\prime}\left[\mu_{n}^{\eta, \beta}\left[\beta H_{n}^{\check{\eta}-\eta}\right]\right] \\
& \geq Q\left[\mu_{n}^{\eta, \beta}\left[Q^{\prime}\left[\beta H_{n}^{\zeta}\right]\right]\right] \\
& =n \beta(q-p) .
\end{aligned}
$$

From these estimates, it follows that for any $M>0$,

$$
\begin{aligned}
\lim _{q \downarrow p} \sup _{|\beta| \leq M} & |\varphi(q, \beta)-\varphi(p, \beta)| \\
& =\lim _{q \downarrow p} \sup _{|\beta| \leq M} \lim _{n \rightarrow \infty} \frac{1}{n}\left|Q \otimes Q^{\prime}\left[\log Z_{n}^{\check{\eta}, \beta}-\log Z_{n}^{\eta, \beta}\right]\right| \\
& =0
\end{aligned}
$$

and the same holds for $\lim _{p \uparrow q}$. Combining with the continuity in $\beta$, we get the joint continuity on $(p, \beta) \in(0,1] \times \mathbb{R}$.

Now we proceed to the main part of the proof, that is, the continuity at $\beta=-\infty$. The following is the key estimate.

Proposition 1. Let $\alpha \in(0, d), p \in(0,1)$ and $\epsilon>0$. Then there exist $r>0$ and $\beta_{0}<0$ such that for all $q \in[p, p+r]$ and $\beta \in\left[-\infty, \beta_{0}\right]$, Q-almost surely for all sufficiently large $n$,

$$
Z_{n}^{\eta, \beta} \leq e^{\epsilon n} Z_{n}^{\check{\eta},-\infty}
$$

Let us first see how to derive Theorem 1 from this proposition. Since the other direction $Z_{n}^{\eta, \beta} \geq Z_{n}^{\check{\eta},-\infty}$ is obvious, we see that

$$
\begin{aligned}
\varphi(p, \beta)-\epsilon & \leq \liminf _{n \rightarrow \infty} \frac{1}{n} \log Z_{n}^{\check{\eta},-\infty} \\
& \leq \limsup _{n \rightarrow \infty} \frac{1}{n} \log Z_{n}^{\check{\eta},-\infty} \leq \varphi(p, \beta) .
\end{aligned}
$$


This in particular implies (by setting $q=p$ ) that the limit (1.4) exists and equals to $\lim _{\beta \rightarrow-\infty} \varphi(p, \beta)$. Thus, (2.3) reads:

$$
\varphi(p, \beta)-\epsilon \leq \varphi(q,-\infty) \leq \varphi(p, \beta) .
$$

Therefore, it also follows from the monotonicity and (2.4) that

$$
\begin{aligned}
& \sup \left\{\left|\varphi\left(p_{1}, \beta_{1}\right)-\varphi\left(p_{2}, \beta_{2}\right)\right|: p_{1}, p_{2} \in[p, p+r], \beta_{1}, \beta_{2} \in\left[\beta_{0},-\infty\right]\right\} \\
& \quad \leq \varphi\left(p, \beta_{0}\right)-\varphi(p+r,-\infty) \\
& \quad \leq 2 \epsilon .
\end{aligned}
$$

This, together with (2.1), completes the proof of the joint continuity.

Proposition 1. Hereafter, we denote $Q \otimes Q^{\prime}$ by $Q$ for simplicity. The basic strategy of the proof is to deform the path appearing in the sum

$$
Z_{n}^{\eta, \beta}=\sum_{x_{1}, \ldots, x_{n}} \prod_{j=1}^{n} f\left(\left|x_{j-1}-x_{j}\right|_{1}\right) e^{\beta \eta\left(j, x_{j}\right)}
$$

to a path $x^{*}$ which does not hit a site with $\check{\eta}(j, x)=1$ and compare the above with

$$
\sum_{x_{1}^{*}, \ldots, x_{n}^{*}} \prod_{j=1}^{n} f\left(\left|x_{j-1}^{*}-x_{j}^{*}\right|_{1}\right) \leq Z_{n}^{\eta,-\infty},
$$

where the sum runs over all paths which appear as a result of deformation. To establish (2.2), we need

(i) the deformation costs $\prod_{j=1}^{n} \frac{f\left(\left|x_{j-1}-x_{j}\right|_{1}\right)}{f\left(\left|x_{j-1}^{*}-x_{j}^{*}\right|_{1}\right)}$ are negligible;

(ii) not too many paths are deformed to a single path $x^{*}$.

Let us start the proper proof. We define $x^{*}$ as follows:

$$
x_{k}^{*}= \begin{cases}x_{k}, & \text { if } \check{\eta}\left(k, x_{k}\right)=0, \\ \operatorname{argmin}\left\{\operatorname{dist}_{1}(x,\{x: \check{\eta}(k, x)=0\})\right\}, & \text { if } \check{\eta}\left(k, x_{k}\right)=1,\end{cases}
$$

where if there are several candidates in the second case, we choose one by a deterministic algorithm. To control the costs of deformation, we define

$$
d_{j}\left(X_{j}, \check{\eta}\right)=\operatorname{dist}_{1}\left(X_{j},\{x: \check{\eta}(j, x)=0\}\right),
$$

where dist ${ }_{1}$ denotes the $l^{1}$-distance, and introduce an auxiliary Hamiltonian

$$
D_{n}(X, \check{\eta})=\sum_{j=1}^{n} d_{j}\left(X_{j}, \check{\eta}\right)^{\alpha}
$$

for $\alpha<1$ and

$$
D_{n}(X, \check{\eta})=\sum_{j=1}^{n} d_{j}\left(X_{j}, \check{\eta}\right)^{\alpha}+\left|X_{j-1}-X_{j}\right|_{1}^{\alpha-1}\left(d_{j-1}\left(X_{j-1}, \check{\eta}\right)+d_{j}\left(X_{j}, \check{\eta}\right)\right)
$$


for $1 \leq \alpha<d$ with the convention $d_{0}\left(X_{0}, \check{\eta}\right)=0$. When $\alpha<1$, we use the fact $(x+y)^{\alpha} \leq x^{\alpha}+y^{\alpha}$ for positive $x, y$ to bound the deformation cost at each step as

$$
\begin{aligned}
\frac{f\left(\left|x_{j-1}-x_{j}\right|_{1}\right)}{f\left(\left|x_{j-1}^{*}-x_{j}^{*}\right|_{1}\right)} & =\exp \left\{c_{2}\left(\left|x_{j-1}^{*}-x_{j}^{*}\right|_{1}^{\alpha}-\left|x_{j-1}-x_{j}\right|_{1}^{\alpha}\right)\right\} \\
& \leq \exp \left\{c_{2}\left(\left|x_{j-1}-x_{j-1}^{*}\right|_{1}^{\alpha}+\left|x_{j}-x_{j}^{*}\right|_{1}^{\alpha}\right)\right\}
\end{aligned}
$$

In the other case $1 \leq \alpha<d$, we instead use convexity to get

$$
\begin{aligned}
\left|x_{j-1}^{*}-x_{j}^{*}\right|_{1}^{\alpha}-\left|x_{j-1}-x_{j}\right|_{1}^{\alpha} & \leq\left[\left|x_{j-1}-x_{j}\right|_{1}+d_{j-1}\left(x_{j-1}, \check{\eta}\right)+d_{j}\left(x_{j}, \check{\eta}\right)\right]^{\alpha}-\left|x_{j-1}-x_{j}\right|_{1}^{\alpha} \\
\quad \leq & \alpha\left[\left|x_{j-1}-x_{j}\right|_{1}+d_{j-1}\left(x_{j-1}, \check{\eta}\right)+d_{j}\left(x_{j}, \check{\eta}\right)\right]^{\alpha-1}\left(d_{j-1}\left(x_{j-1}, \check{\eta}\right)+d_{j}\left(x_{j}, \check{\eta}\right)\right) \\
& \leq \alpha 2^{\alpha}\left|x_{j-1}-x_{j}\right|_{1}^{\alpha-1}\left(d_{j-1}\left(x_{j-1}, \check{\eta}\right)+d_{j}\left(x_{j}, \check{\eta}\right)\right) \\
& \quad+\alpha 2^{2 \alpha}\left(d_{j-1}\left(x_{j-1}, \check{\eta}\right)^{\alpha}+d_{j}\left(x_{j}, \check{\eta}\right)^{\alpha}\right) .
\end{aligned}
$$

Hence in both cases, the total cost is bounded as

$$
\prod_{j=1}^{n} \frac{f\left(\left|x_{j-1}-x_{j}\right|_{1}\right)}{f\left(\left|x_{j-1}^{*}-x_{j}^{*}\right|_{1}\right)} \leq e^{c_{3} D_{n}}
$$

for some $c_{3}>0$.

Lemma 1. Let $\alpha \in(0, d)$. For any $p \in(0,1)$ and $\delta>0$, there exists $r \in(0,1)$ and $\beta_{0}<0$ such that for all $q \in[p, p+r]$ and $\beta \leq \beta_{0}$,

$$
\lim _{n \rightarrow \infty} \frac{1}{Z_{n}^{\eta, \beta}} P\left[\exp \left\{\beta H_{n}^{\eta}\right\}: D_{n} \leq \delta n\right]=1, \quad \text { Q-a.s. }
$$

Proof. We give a proof only in the case $1 \leq \alpha<d$ since the other case is easier. We show that for any $\gamma>0$, one can find $\beta_{0}$ and $r$ such that

$$
Q\left[P\left[\exp \left\{\beta H_{n}^{\eta}+\gamma D_{n}\right\}\right]\right] \leq 1
$$

for all $q \in[p, p+r]$ and $\beta \leq \beta_{0}$. Then it readily follows that $Q$-almost surely,

$$
P\left[\exp \left\{\beta H_{n}^{\eta}+\gamma D_{n}\right\}\right] \leq n^{2}
$$

except for finitely many $n \in N$. If we take $\gamma>\lim _{\beta \rightarrow-\infty}|\varphi(p, \beta)| / \delta$, the right-hand side of

$$
P\left[\exp \left\{\beta H_{n}^{\eta}\right\}: D_{n}>\delta n\right] \leq e^{-\gamma \delta n} P\left[\exp \left\{\beta H_{n}^{\eta}+\gamma D_{n}\right\}\right]
$$

is $o\left(Z_{n}^{\eta, \beta}\right)$ and we are done. 
Let us fix an arbitrary $\gamma>0$ and we write

$$
\begin{aligned}
& Q\left[P\left[\exp \left\{\beta H_{n}^{\eta}+\gamma D_{n}\right\}\right]\right] \\
& =P\left[\prod _ { j = 1 } ^ { n } Q \left[\operatorname { e x p } \left\{\beta \eta\left(j, X_{j}\right)+\gamma d_{j}\left(X_{j}, \check{\eta}\right)^{\alpha}\right.\right.\right. \\
& \left.\left.\left.\quad+\gamma\left(\left|X_{j-1}-X_{j}\right|_{1}^{\alpha-1}+\left|X_{j}-X_{j+1}\right|_{1}^{\alpha-1}\right) d_{j}\left(X_{j}, \check{\eta}\right)\right\}\right]\right]
\end{aligned}
$$

with the convention $\left|X_{n}-X_{n+1}\right|_{1}=0$. We estimate the last $Q$-expectation by distinguishing the cases according to the value of $\check{\eta}\left(j, X_{j}\right)$. First, if $\check{\eta}\left(j, X_{j}\right)=0$ then all terms in the exponential are zero and, by definition,

$$
Q\left(\check{\eta}\left(j, X_{j}\right)=0\right)=1-q
$$

Second since $\eta\left(j, X_{j}\right)$ and $d_{j}\left(X_{j}, \check{\eta}\right)$ are conditionally independent on $\left\{\check{\eta}\left(j, X_{j}\right)=1\right\}$, we get for general $\xi>0$,

$$
\begin{aligned}
Q & {\left[e^{\beta \eta\left(j, X_{j}\right)+\xi d_{j}\left(X_{j}, \check{\eta}\right)} 1_{\left\{\check{\eta}\left(j, X_{j}\right)=1\right\}}\right] } \\
& =Q\left[e^{\beta \eta\left(j, X_{j}\right)} 1_{\left\{\check{\eta}\left(j, X_{j}\right)=1\right\}}\right] Q\left[e^{\xi d_{j}\left(X_{j}, \check{\eta}\right)} \mid \check{\eta}\left(j, X_{j}\right)=1\right] \\
& \leq \delta(\beta, r) Q\left[e^{\xi d_{j}\left(X_{j}, \check{\eta}\right)} \mid \check{\eta}\left(j, X_{j}\right)=1\right]
\end{aligned}
$$

where $\delta(\beta, r)=e^{\beta}+r \geq e^{\beta}+Q\left(\eta\left(j, X_{j}\right)=0, \zeta\left(j, X_{j}\right)=1\right)$. The upper tail of the distribution of $d_{j}\left(X_{j}, \check{\eta}\right)$ under $Q\left(\cdot \mid \check{\eta}\left(j, X_{j}\right)=1\right)$ is bounded as

$$
\begin{aligned}
& Q\left(d_{j}\left(X_{j}, \check{\eta}\right)>r \mid \check{\eta}\left(j, X_{j}\right)=1\right) \\
& \quad=Q\left(\check{\eta}(j, x)=1 \text { for } 1 \leq\left|x-X_{j}\right|_{1} \leq r\right) \\
& \quad \leq q^{c r^{d}}
\end{aligned}
$$

As a consequence, we obtain

$$
Q\left[e^{\beta \eta\left(j, X_{j}\right)+\xi d_{j}\left(X_{j}, \check{\eta}\right)}\right] \leq 1-q+\delta(\beta, r) e^{\Lambda(\xi)}
$$

for some regularly varying function $\Lambda$ of index $d /(d-1)$ by a standard Tauberian argument. (See, for example, [23]. In fact, it is easy to check this fact directly by a Laplace principle type argument.) Similarly it also follows from the assumption $\alpha<d$ that

$$
Q\left[e^{\beta \eta\left(j, X_{j}\right)+\xi d_{j}\left(X_{j}, \check{\eta}\right)^{\alpha}}\right]<1-q+\delta(\beta, r) \Theta(\xi)
$$

for some $\Theta(\xi)<\infty$. 
Now we rewrite the exponential in (2.8) as

$$
\begin{aligned}
& \exp \left\{\frac{\beta}{3} \eta\left(j, X_{j}\right)+\gamma d_{j}\left(X_{j}, \check{\eta}\right)^{\alpha}\right\} \\
& \exp \left\{\frac{\beta}{3} \eta\left(j, X_{j}\right)+\gamma d_{j}\left(X_{j}, \check{\eta}\right)\left|X_{j-1}-X_{j}\right|_{1}^{\alpha-1}\right\} \\
& \quad \exp \left\{\frac{\beta}{3} \eta\left(j, X_{j}\right)+\gamma d_{j}\left(X_{j}, \check{\eta}\right)\left|X_{j}-X_{j+1}\right|_{1}^{\alpha-1}\right\}
\end{aligned}
$$

and apply Hölder's inequality and (2.9) to obtain

$$
\begin{aligned}
Q\left[\operatorname { e x p } \left\{\beta \eta\left(j, X_{j}\right)+\gamma d_{j}\left(X_{j}, \check{\eta}\right)^{\alpha}\right.\right. & \\
& \left.\left.+\gamma d_{j}\left(X_{j}, \check{\eta}\right)\left(\left|X_{j-1}-X_{j}\right|_{1}^{\alpha-1}+\left|X_{j}-X_{j+1}\right|_{1}^{\alpha-1}\right)\right\}\right] \\
\leq & (1-q+\delta(\beta, r) \Theta(3 \gamma))^{1 / 3} \\
& \left(1-q+\delta(\beta, r) e^{\Lambda\left(3 \gamma\left|X_{j-1}-X_{j}\right|_{1}^{\alpha-1}\right)}\right)^{1 / 3} \\
& \left(1-q+\delta(\beta, r) e^{\Lambda\left(3 \gamma\left|X_{j}-X_{j+1}\right|_{1}^{\alpha-1}\right)}\right)^{1 / 3}
\end{aligned}
$$

We may drop the first factor on the right-hand side since it can be made smaller than one by letting $\beta$ be close to $-\infty$ and $r$ close to zero. We then take the product over $1 \leq j \leq n$ and $P$-expectation. Due to the independence of $\left\{X_{j-1}-X_{j}\right\}_{j=1}^{n}$ under $P$, the expectation factorizes and the term containing $X_{j-1}-X_{j}$ is

$$
\begin{aligned}
& P\left[\left(1-q+\delta(\beta, r) e^{\Lambda\left(3 \gamma\left|X_{j-1}-X_{j}\right|_{1}^{\alpha-1}\right)}\right)^{2 / 3}\right] \\
& \quad \stackrel{\text { Jensen }}{\leq}\left(1-q+\delta(\beta, r) P\left[e^{\Lambda\left(3 \gamma\left|X_{j-1}-X_{j}\right|_{1}^{\alpha-1}\right)}\right]\right)^{2 / 3}
\end{aligned}
$$

for $2 \leq j \leq n-1$ and for $j \in\{1, n\}$, the exponent $2 / 3$ is replaced by $1 / 3$. In this way, our problem is reduced to checking that

$$
P\left[e^{\Lambda\left(3 \gamma\left|X_{j-1}-X_{j}\right|_{1}^{\alpha-1}\right)}\right]<\infty
$$

But the function $x \mapsto \Lambda\left(3 \gamma x^{\alpha-1}\right)$ is regularly varying of index $(\alpha-1) \frac{d}{d-1}<\alpha$ for $\alpha<d$, hence the above expectation is finite. 
Due to the above lemma, we can restrict the summation (2.5) to paths with $D_{n}(x, \check{\eta}) \leq \delta n$ and get

$$
\begin{aligned}
Z_{n}^{\eta, \beta} & \sim \sum_{x_{1}, \ldots, x_{n}: D_{n}(x, \check{\eta}) \leq \delta n} \prod_{j=1}^{n} f\left(\left|x_{j-1}-x_{j}\right|_{1}\right) e^{\beta \eta\left(j, x_{j}\right)} \\
& =\sum_{x_{1}, \ldots, x_{n}: D_{n}(x, \check{\eta}) \leq \delta n} \prod_{j=1}^{n} f\left(\left|x_{j-1}^{*}-x_{j}^{*}\right|_{1}\right)\left[\frac{f\left(\left|x_{j-1}-x_{j}\right|_{1}\right)}{f\left(\left|x_{j-1}^{*}-x_{j}^{*}\right|_{1}\right)} e^{\beta \eta\left(j, x_{j}\right)}\right] \\
& \leq e^{c_{3} \delta n} \sum_{y_{1}, \ldots, y_{n}: H_{n}(y, \check{\eta})=0} \#\left\{x: x^{*}=y, D_{n}(x, \check{\eta}) \leq \delta n\right\} \prod_{j=1}^{n} f\left(\left|y_{j-1}-y_{j}\right|_{1}\right) .
\end{aligned}
$$

We are left with estimating the number of paths which are deformed to a fixed path.

Lemma 2. There exists a function $\chi(\delta) \rightarrow 0$ as $\delta \downarrow 0$ such that for any fixed path $\left(y_{1}, \ldots, y_{n}\right) \in\left(\mathbb{Z}^{d}\right)^{n}$,

$$
\#\left\{x: x^{*}=y, D_{n}(x, \check{\eta}) \leq \delta n\right\} \leq \exp \{\chi(\delta) n\} .
$$

Proof. We write $z_{j}=x_{j}-y_{j}$. Then it suffices to bound

$$
\begin{aligned}
& \#\left\{\left(z_{j}\right)_{j=1}^{n}:\left|z_{1}\right|_{1}^{\alpha}+\cdots+\left|z_{n}\right|_{1}^{\alpha} \leq \delta n\right\} \\
& \quad \leq e^{\lambda \delta n} \sum_{z:\left|z_{1}\right|_{1}^{\alpha}+\cdots+\left|z_{n}\right|_{1}^{\alpha} \leq \delta n} e^{-\lambda\left(\left|z_{1}\right|_{1}^{\alpha}+\cdots+\left|z_{n}\right|_{1}^{\alpha}\right)} \quad(\lambda>0) \\
& \quad \leq\left(\sum_{z \in \mathbb{Z}^{d}} e^{\lambda \delta-\lambda|z|_{1}^{\alpha}}\right)^{n} .
\end{aligned}
$$

By taking $\lambda=\delta^{-1 / 2}$, we find that the right-hand side is $(1+o(1))^{n}$ as $\delta \downarrow 0$.

Combining the above arguments, we can find $r \in(0,1)$ and $\beta_{0}<0$ such that for any $q \in[p, p+r]$ and $\beta<\beta_{0}$,

$$
\begin{aligned}
Z_{n}^{\eta, \beta} & \leq e^{\epsilon n} \sum_{y_{1}, \ldots, y_{n}: D_{n}(y, \check{\eta})=0} \prod_{k=1}^{n} f\left(\left|y_{j-1}-y_{j}\right|_{1}\right) \\
& =e^{\epsilon n} Z_{n}^{\check{\eta},-\infty}
\end{aligned}
$$

for all sufficiently large $n \in \mathbb{N}$.

\section{A DIRECTED FIRST PASSAGE PERCOlation}

In this section, we prove Theorem 3 . We also prove a concentration bound for the passage times, which is an important ingredient in the proof of Theorem 2.

For further use, we start by introducing a special realization of $\eta$ : recalling that $\eta=\eta_{p}$ depends in fact on $p$, we define a coupling of $\eta_{p}$ for all values of $p \in(0,1)$ as 
follows. Let $\left(Q, \omega_{1}\right)$ be the Poisson point process on $\mathbb{N} \times \mathbb{R}^{d}$ whose intensity is the product of the counting measure and Lebesgue measure, and define, for $p \in(0,1)$,

$$
\eta(k, x)=\eta_{p}(k, x)=1_{\left\{\omega_{1}\left(\{k\} \times s_{p}\left(x+[0,1)^{d}\right)\right)=0\right\}}
$$

with $s_{p}=\left(\log \frac{1}{p}\right)^{1 / d}$ the scaling factor. Note that $s_{p} \in(0, \infty)$ and $s_{p} \rightarrow 0$ as $p \uparrow 1$. Let us also introduce

$$
\omega_{p}=\sum_{(k, x) \in \mathbb{N} \times \mathbb{Z}^{d}}\left(1-\eta_{p}(k, x)\right) \delta_{\left(k, s_{p} x\right)}
$$

which vaguely converges to $\omega_{1}, Q$-almost surely as $p \uparrow 1$. Hereafter, we sometimes identify $\omega_{p}$ with its support by abuse of notation. For $0<p \leq 1$, recall the definition of the passage time from 0 to $n$,

$$
T_{n}\left(\omega_{p}\right)=\min \left\{\sum_{k=1}^{n}\left|x_{k-1}-x_{k}\right|^{\alpha}: x_{0}=0 \text { and }\left\{\left(k, x_{k}\right)\right\}_{k=1}^{n} \subset \omega_{p}\right\},
$$

and recall that, by the subadditive ergodic theorem, the following limits exist and are equal:

$$
\mu_{p}=\text { a.s.- } \lim _{n \rightarrow \infty} \frac{1}{n} T_{n}\left(\omega_{p}\right)=\inf _{n \in \mathbb{N}} \frac{1}{n} Q\left[T_{n}\left(\omega_{p}\right)\right]=\lim _{n \rightarrow \infty} \frac{1}{n} Q\left[T_{n}\left(\omega_{p}\right)\right] .
$$

Theorem 3. We have the following comparison for the passage times from which the result readily follows:

$$
\begin{aligned}
& T_{n}\left(\omega_{1}\right) \leq\left(1+\delta_{1}\right) T_{n}\left(\omega_{p}\right)+\delta_{2} n, \\
& T_{n}\left(\omega_{p}\right) \leq\left(1+\delta_{1}\right) T_{n}\left(\omega_{1}\right)+\delta_{2} n,
\end{aligned}
$$

where $\delta_{1}, \delta_{2} \rightarrow 0$ as $p \uparrow 1$. We only prove the first one since the argument for the other is the same. Let $\left(\pi_{n}(m)\right)_{m=0}^{n}$ be a minimizing path for $T_{n}\left(\omega_{p}\right)$. Then, by definition, each $\pi_{n}(m)+\left[0, s_{p}\right)^{d}$ contains a point of $\omega_{1}$. Thus we can find another path $\left\{\pi_{n}^{\prime}(m)\right\}_{m=0}^{n}$ such that

$$
\pi_{n}^{\prime}(0)=0, \pi_{n}^{\prime}(m) \in \omega_{1} \text { and }\left|\pi_{n}(m)-\pi_{n}^{\prime}(m)\right|_{1} \leq d s_{p}
$$

for $1 \leq m \leq n$. Then, we have

$$
\left|\pi_{n}^{\prime}(m-1)-\pi_{n}^{\prime}(m)\right|_{1} \leq\left|\pi_{n}(m-1)-\pi_{n}(m)\right|_{1}+2 d s_{p}
$$

and together with an elementary inequality

$$
(t+s)^{\alpha} \leq \begin{cases}t^{\alpha}+s^{\alpha}, & \alpha \leq 1 \\ (1+s)^{\alpha-1}\left(t^{\alpha}+s\right), & \alpha>1\end{cases}
$$


where the second one is obtained by applying convexity to $\left(\frac{1 \cdot t+s \cdot 1}{1+s}\right)^{\alpha}$, we get

$$
\begin{aligned}
T_{n}\left(\omega_{1}\right) & \leq \sum_{m=1}^{n}\left|\pi_{n}^{\prime}(m-1)-\pi_{n}^{\prime}(m)\right|_{1}^{\alpha} \\
& \leq \begin{cases}\sum_{m=1}^{n}\left|\pi_{n}(m-1)-\pi_{n}(m)\right|_{1}^{\alpha}+\left(2 d s_{p}\right)^{\alpha} n, & \alpha \leq 1, \\
\left(1+2 d s_{p}\right)^{\alpha-1}\left(\sum_{m=1}^{n}\left|\pi_{n}(m-1)-\pi_{n}(m)\right|_{1}^{\alpha}+2 d s_{p} n\right), & \alpha>1 .\end{cases}
\end{aligned}
$$

Since $s_{p}$ tends to zero as $p \uparrow 1$, we are done.

Our second main result in this subsection is the lower tail estimate of the passage time distribution.

Proposition 2. There exist positive constants $C_{1}, C_{2}$ and $\lambda \in(0,1)$ such that for any $n \in \mathbb{N}$,

$$
Q\left(T_{n}\left(\omega_{1}\right)-n \mu_{1}<-n^{1-\lambda}\right) \leq C_{1} \exp \left\{-C_{2} n^{\lambda}\right\} .
$$

Proof. We fix a small $\theta>0$ and define

$$
\bar{\omega}=\omega+\sum_{(k, x) \in \mathbb{N} \times n^{\theta} \mathbb{Z}^{d}} 1_{\left\{\omega\left(\{k\} \times\left(x+\left[0, n^{\theta}\right)^{d}\right)\right)=0\right\}} \delta_{(k, x)},
$$

that is, when we find a large vacant box, we add an $\omega$-point artificially at a corner. This modification provides a uniform bound for the passage time

$$
\sup _{\omega} T_{n}(\bar{\omega}) \leq d^{\alpha} n^{1+\alpha \theta}
$$

since there is a path whose all jumps are bounded by $d n^{\theta}$. We also have the following upper tail estimate.

Lemma 3. There exists $C_{0}>0$ such that for all $n \in \mathbb{N}$ and $m>C_{0} n$,

$$
Q\left(T_{n}\left(\omega_{1}\right)>m\right) \leq \exp \left\{-m^{1 \wedge \frac{d}{\alpha}} / C_{0}\right\} .
$$

Proof. Note that $T_{n}\left(\omega_{1}\right)$ is bounded by the passage time of the greedy path $\left\{\left(k, x_{k}\right)\right\}_{k \in \mathbb{N}}$ which is inductively constructed by minimizing the distance to points in the next section, that is, $x_{0}=0$ and

$$
x_{k}=\operatorname{argmin}\left\{\left|x_{k-1}-x\right|_{1}:(k, x) \in \omega_{1}\right\} .
$$

The passage time of such a path is nothing but the sum of independent random variables with the same distribution as $\operatorname{dist}\left((0,0),\left.\omega_{1}\right|_{\{0\} \times \mathbb{R}^{d}}\right)^{\alpha}$. One can bound its tail as

$$
\begin{aligned}
Q\left(\operatorname{dist}\left((0,0),\left.\omega_{1}\right|_{\{0\} \times \mathbb{R}^{d}}\right)^{\alpha} \geq r\right) & \left.=Q\left(\left.\omega_{1}\right|_{\{0\} \times \mathbb{R}^{d}}\left(B_{l^{1}}\left(0, r^{1 / \alpha}\right)\right)=0\right)\right) \\
& =\exp \left\{-c r^{d / \alpha}\right\}
\end{aligned}
$$

for some $c>0$. Our assertion follows from this and a well known result for the large deviation of sums of independent random variables, for which we refer to [29].

Next, we show that $T_{n}\left(\omega_{1}\right)$ and $T_{n}\left(\bar{\omega}_{1}\right)$ are essentially the same. 
Lemma 4. There exists $C_{3}>0$ such that for sufficiently large $n \in \mathbb{N}$,

$$
\begin{gathered}
\max \left\{Q\left(T_{n}\left(\omega_{1}\right) \neq T_{n}\left(\bar{\omega}_{1}\right)\right), Q\left[\left|T_{n}\left(\omega_{1}\right)-T_{n}\left(\bar{\omega}_{1}\right)\right|\right]\right\} \\
\leq \exp \left\{-C_{3} n^{d \theta}\right\} .
\end{gathered}
$$

Proof. Thanks to Lemma 3, we know that $T_{n}\left(\omega_{1}\right) \leq C_{0} n$ with probability greater than $1-\exp \left\{-n^{1 \wedge \frac{d}{\alpha}} / C_{0}\right\}$. Under this condition, all the minimizing paths for $T_{n}\left(\omega_{1}\right)$ stay inside $\mathcal{C}_{n}:=[0, n] \times\left[-C_{0}^{1 / \alpha} n^{1+1 / \alpha}, C_{0}^{1 / \alpha} n^{1+1 / \alpha}\right]^{d}$. Indeed, if any minimizing path exits $\mathcal{C}_{n}$, then it must make a jump larger than $C_{0}^{1 / \alpha} n^{1 / \alpha}$ and hence its passage time is larger than $C_{0} n$. Since $T_{n}\left(\bar{\omega}_{1}\right) \leq T_{n}\left(\omega_{1}\right)$, the same applies to minimizing paths for $T_{n}\left(\bar{\omega}_{1}\right)$. This space-time region contains only polynomially many boxes of the form $\{k\} \times\left(x+\left[0, n^{\theta}\right)^{d}\right)$ and each of them is vacant with probability $\exp \left\{-c n^{d \theta}\right\}$. Thus it follows that

$$
Q\left(\omega_{1}=\bar{\omega}_{1} \text { in } \mathcal{C}_{n}\right) \geq 1-\exp \left\{-c n^{d \theta} / 2\right\}
$$

for large $n$. Since $T_{n}\left(\omega_{1}\right)=T_{n}\left(\bar{\omega}_{1}\right)$ on the event

$$
\left\{T_{n}\left(\omega_{1}\right) \leq C_{0} n \text { and } \omega_{1}=\bar{\omega}_{1} \text { in } \mathcal{C}_{n}\right\}
$$

we get the desired bound on $Q\left(T_{n}\left(\omega_{1}\right) \neq T_{n}\left(\bar{\omega}_{1}\right)\right)$.

As for the $L^{1}(Q)$ distance, we use the Schwarz inequality to obtain

$$
\begin{aligned}
& Q\left[\left|T_{n}\left(\omega_{1}\right)-T_{n}\left(\bar{\omega}_{1}\right)\right|\right] \\
& \quad \leq Q\left[\left(T_{n}\left(\omega_{1}\right)-T_{n}\left(\bar{\omega}_{1}\right)\right)^{2}\right]^{1 / 2} Q\left(T_{n}\left(\omega_{1}\right) \neq T_{n}\left(\bar{\omega}_{1}\right)\right)^{1 / 2} .
\end{aligned}
$$

The first factor on the right-hand side is of $O(n)$ as $n \rightarrow \infty$ due to Lemma 3 .

We proceed to a lower tail estimate for $T_{n}\left(\bar{\omega}_{1}\right)$. Let $\bar{\omega}_{1}^{(m)}$ be the point process obtained by replacing its $\{m\} \times \mathbb{R}^{d}$-section by $\bar{\omega}^{\prime}$ which is the modification of another configuration $\omega^{\prime}$. We are going to use the so-called entropy method (Theorem 6.7 in [2]) and it requires a bound on

$$
\sum_{m=1}^{n}\left(\sup _{\omega^{\prime}} T_{n}\left(\bar{\omega}_{1}^{(m)}\right)-T_{n}\left(\bar{\omega}_{1}\right)\right)^{2} .
$$

Let us first assume $\alpha \geq 1$ and let $\left\{\pi_{n}(m)\right\}_{m=0}^{n}$ be a minimizing path for $T_{n}\left(\bar{\omega}_{1}\right)$. As we can find a point in $\left.\omega^{\prime}\right|_{\{m\} \times \mathbb{R}^{d}}$ within the distance $d n^{\theta}$ to $\pi_{n}(m)$,

$$
\begin{aligned}
\sup _{\omega^{\prime}} & T_{n}\left(\bar{\omega}_{1}^{(m)}\right)-T_{n}\left(\bar{\omega}_{1}\right) \\
\leq & \alpha\left(\left|\pi_{n}(m-1)-\pi_{n}(m)\right|_{1}+d n^{\theta}\right)^{\alpha-1} d n^{\theta} 1_{\{m \geq 1\}} \\
& +\alpha\left(\left|\pi_{n}(m)-\pi_{n}(m+1)\right|_{1}+d n^{\theta}\right)^{\alpha-1} d n^{\theta} 1_{\{m \leq n-1\}} .
\end{aligned}
$$

Furthermore, the a priori bound

$$
T_{n}\left(\bar{\omega}_{1}\right)=\sum_{m=1}^{n}\left|\pi_{n}(m-1)-\pi_{n}(m)\right|_{1}^{\alpha} \leq d^{\alpha} n^{1+\alpha \theta}
$$


yields the following bound on the numbers of large jumps

$$
\#\left\{m \leq n:\left|\pi_{n}(m-1)-\pi_{n}(m)\right|_{1} \geq n^{k \theta}\right\} \leq d^{\alpha} n^{1-(k-1) \alpha \theta} 1_{\left\{k \leq \frac{1}{\alpha \theta}+2\right\}} .
$$

Thus by dividing the sum in (3.5) according to the indices with jump size falling in $\left[n^{k \theta}, n^{(k+1) \theta}\right)$, we can bound it, up to a multiplicative constant, by

$$
\sum_{k \leq \frac{1}{\alpha \theta}+2} n^{1-(k-1) \alpha \theta} n^{2(k+1) \theta(\alpha-1)+2 \theta}=n^{1+3 \alpha \theta} \sum_{k \leq \frac{1}{\alpha \theta}+2} n^{(\alpha-2) \theta k} .
$$

It is simple to check that the right-hand side is bounded by $n^{\rho}$ with $\rho<2$ when $\theta$ is sufficiently small. Then, Theorem 6.7 in [2] yields

$$
Q\left(T_{n}\left(\bar{\omega}_{1}\right)-Q\left[T_{n}\left(\bar{\omega}_{1}\right)\right]<-n^{1-\lambda}\right) \leq \exp \left\{-C_{2} n^{2-\rho-2 \lambda}\right\} .
$$

Lemma 4 shows that this remains valid with $\bar{\omega}_{1}$ replaced by $\omega_{1}$ and $\exp \left\{-C_{3} n^{d \theta}\right\}$ added to the right-hand side. Finally, since $\mu_{1}=\inf _{n} n^{-1} Q\left[T_{n}\left(\omega_{1}\right)\right]$, we can further replace $Q\left[T_{n}\left(\omega_{1}\right)\right]$ by $n \mu_{1}$ and arrive at

$$
Q\left(T_{n}\left(\omega_{1}\right)-n \mu_{1}<-n^{1-\lambda}\right) \leq \exp \left\{-C_{2} n^{2-\rho-2 \lambda}\right\}+\exp \left\{-C_{3} n^{d \theta}\right\} .
$$

Choosing $\lambda>0$ small, we get the desired bound.

The case $\alpha<1$ is simpler since we readily get $\sup _{\omega^{\prime}} T_{n}\left(\bar{\omega}_{1}^{(m)}\right)-T_{n}\left(\bar{\omega}_{1}\right) \leq 2 d^{\alpha} n^{\alpha \theta}$ uniformly in $m$ just as in (2.6).

\section{Proof of Theorem 2}

In this section, we continue to assume that $\eta$ is realized as in (3.1) in the previous section. Recall also that we defined $s_{p}=\left(\log \frac{1}{p}\right)^{1 / d}$, which is asymptotic to $(1-p)^{1 / d}$ as $p \uparrow 1$. The positivity of $\mu_{1}$ can proved by essentially the same argument as in the upper bound: see Remark 4 below. Let us first complete the proof of (1.5) assuming it.

Lower bound. Let $\pi_{n}$ be a minimizing path for $T_{n}\left(\omega_{p}\right)$. Then obviously,

$$
\begin{aligned}
Z_{n}^{\eta,-\infty} & =P\left(H_{n}^{\eta}=0\right) \\
& \geq P\left(X_{k}=\pi_{n}(k) \text { for all } 1 \leq k \leq n\right) \\
& =c_{1}^{n} \exp \left\{-c_{2} s_{p}^{-\alpha} T_{n}\left(\omega_{p}\right)\right\}
\end{aligned}
$$

and hence

$$
\varphi(p,-\infty) \geq-c_{2} s_{p}^{-\alpha} \mu_{p}+\log c_{1} .
$$

By letting $p \uparrow 1$ and using Theorem 3, we get the desired lower bound.

The upper bound is more laborious since we have to show that the number of paths makes negligible contribution. We closely follow the argument of Mountford in [28]. 
Upper bound. Let $M=(\alpha+2) / \alpha$ and define a face-to-face passage time

$$
\Phi_{R}\left(\omega_{p}\right)=\inf \left\{\sum_{i=1}^{R}\left|x_{i-1}-x_{i}\right|_{1}^{\alpha}:\left|x_{0}\right|_{\infty} \leq R^{M} \text { and }\left(i, x_{i}\right) \in \omega_{p} \text { for } 1 \leq i \leq R\right\}
$$

for $R \in \mathbb{N}$. We fix $\epsilon>0$ and say that $(k, x) \in \mathbb{N} \times 2 \mathbb{Z}^{d}$ is $\epsilon$-good if the following two conditions hold:

(i) $\Phi_{R}\left(\omega_{p}-\left(k, R^{M} x\right)\right) \geq\left(\mu_{1}-\epsilon\right) R$;

(ii) $\max _{k+1 \leq l \leq k+R} \omega_{p}\left(\{l\} \times\left(R^{M} x+\left[-2 R^{M}, 2 R^{M}\right]^{d}\right)\right) \leq 4^{d+1} R^{d M}$,

where $\omega_{p}-\left(k, R^{M} x\right)$ is the translation of $\omega_{p}$ regarded as a set. Our basic strategy is to show that: (1) if the polymer, scaled by a factor of $s_{p} R^{-M}$, comes close to an $\epsilon$-good point, then it costs at least $\exp \left\{-\left(\mu_{1}-\epsilon\right) R\right\}$ to survive the next $R$-duration; (2) most of the times in $\{j R\}_{j=1}^{[n / R]}$, the polymer is close to an $\epsilon$-good point with high probability.

Lemma 5. There exists $p_{0}(\epsilon) \in(0,1)$ such that

$$
\lim _{R \rightarrow \infty} Q((k, x) \text { is } \epsilon \text {-good })=1
$$

uniformly in $p \in\left[p_{0}(\epsilon), 1\right]$ and $(k, x) \in \mathbb{N} \times 2 \mathbb{Z}^{d}$.

Proof. By translation invariance, we may assume $(k, x)=(0,0)$ without loss of generality. Note also that the probability of

$$
E_{R}=\left\{\omega_{1}: \max _{y \in\left[-R^{M}, R^{M}\right]^{d} \cap \mathbb{Z}^{d}} T_{R}\left(\omega_{1}-(0, y)\right) \leq C_{0} R\right\}
$$

tends to one as $R \rightarrow \infty$ by Lemma 3. On this event, we know from (3.2) that

$$
T_{R}\left(\omega_{p}-(0, y)\right)<T_{R}\left(\omega_{1}-(0, y)\right)+\epsilon R \leq\left(C_{0}+\epsilon\right) R
$$

for all $p$ close to one. As a consequence, all the minimizing paths for $T_{R}\left(\omega_{p}-(0, y)\right)$, that is, the passage time from $(0, y)$ to $\{R\} \times \mathbb{R}^{d}$, make jumps of size at most a constant multiple of $R^{1 / \alpha}$. Then by using the mean value theorem, one can check that

$$
\Phi_{R}\left(\omega_{p}\right)-\min _{y \in\left[-R^{M}, R^{M}\right]^{d} \cap \mathbb{Z}^{d}} T_{R}\left(\omega_{p}-(0, y)\right) \geq d^{\alpha} \vee\left(c R^{(\alpha-1) / \alpha}\right)
$$

for some $c>0$, since the difference comes only from the starting points.

Thus we can bound

$$
\begin{aligned}
Q( & \left.\left\{\Phi_{R}\left(\omega_{p}\right) \leq\left(\mu_{1}-2 \epsilon\right) R\right\} \cap E_{R}\right) \\
& \leq Q\left(\min _{\left.y \in\left[-R^{M}, R^{M}\right]\right]_{d} \cap \mathbb{Z}^{d}} T_{R}\left(\omega_{1}-(0, y)\right)+d^{\alpha} \vee\left(c R^{(\alpha-1) / \alpha}\right) \leq\left(\mu_{1}-2 \epsilon\right) R\right) \\
& \leq \sum_{y \in\left[-R^{M}, R^{M}\right]^{d} \cap \mathbb{Z}^{d}} Q\left(T_{R}\left(\omega_{1}-(0, y)\right) \leq\left(\mu_{1}-\epsilon\right) R\right) \\
& \leq\left(2 R^{M}+1\right)^{d} C_{1} \exp \left\{-C_{2} R^{\lambda}\right\}
\end{aligned}
$$


for sufficiently large $R$, where we have used (4.2) in the first inequality.

On the other hand, a simple large deviation estimate shows that there is $c>0$ such that for any $l \in \mathbb{N}$,

$$
Q\left(\omega_{p}\left(\{l\} \times\left[-2 R^{M}, 2 R^{M}\right]^{d}\right)>4^{d+1} R^{d M}\right) \leq \exp \left\{-c R^{d M}\right\}
$$

and summing over $l \in\{1,2, \ldots, R\}$, we get

$$
Q\left(\max _{1 \leq l \leq R} \omega_{p}\left(\{l\} \times\left[-2 R^{M}, 2 R^{M}\right]^{d}\right)>4^{d+1} R^{d M}\right) \rightarrow 0
$$

as $R \rightarrow \infty$.

Let us write $C_{p}(x)=s_{p}^{-1}\left(R^{M} x+\left[-R^{M}, R^{M}\right]^{d}\right)$ for shorthand.

Lemma 6. For sufficiently large $R \in \mathbb{N}$, there exists $p_{1}(R, \epsilon)>0$ such that if $p \in\left[p_{1}(R, \epsilon), 1\right)$ and $(k, x)$ is $\epsilon$-good, then

$$
\begin{aligned}
\sup _{y \in C_{p}(x)} P\left(\eta\left(l, X_{l}\right)=\right. & \left.0 \text { for all } l \in\{k+1, \ldots, k+R\} \mid X_{k}=y\right) \\
& \leq \exp \left\{-c_{2} s_{p}^{-\alpha}\left(\mu_{1}-2 \epsilon\right) R\right\} .
\end{aligned}
$$

Proof. We again assume that $(k, x)=(0,0)$ without loss of generality. We first prove

$$
\sup _{y \in C_{p}(0)} P_{y}\left(\max _{1 \leq l \leq R}\left|X_{l}\right|_{\infty} \geq 2 s_{p}^{-1} R^{M}\right) \leq \exp \left\{-C_{5} s_{p}^{-\alpha} R^{2}\right\}
$$

so that we may assume the contrary. When $\alpha \leq 1$, one can readily check that

$$
\begin{aligned}
\sup _{y \in C_{p}(0)} P_{y}\left(\max _{1 \leq l \leq R}\left|X_{l}\right|_{\infty} \geq 2 s_{p}^{-1} R^{M}\right) & \leq P\left(\max _{1 \leq l \leq R}\left|X_{l}\right|_{\infty} \geq s_{p}^{-1} R^{M}\right) \\
& \leq P\left(\sum_{j=1}^{R}\left|X_{j-1}-X_{j}\right|_{1}^{\alpha} \geq s_{p}^{-\alpha} R^{\alpha+2}\right) .
\end{aligned}
$$

Since our assumption on the transition probability implies

$$
C_{6}:=P\left[\exp \left\{\frac{c_{2}}{2}\left|X_{1}\right|_{1}^{\alpha}\right\}\right] \in(1, \infty),
$$

Chebyshev's inequality yields

$$
\text { LHS of }(4.3) \leq \exp \left\{-\frac{c_{2}}{2} s_{p}^{-\alpha} R^{\alpha+2}+R \log C_{6}\right\} .
$$

For $\alpha>1$, we use Jensen's inequality to get

$$
\sup _{y \in C_{p}(0)} P_{y}\left(\max _{1 \leq l \leq R}\left|X_{l}\right|_{\infty} \geq 2 s_{p}^{-1} R^{M}\right) \leq P\left(R^{\alpha-1} \sum_{j=1}^{R}\left|X_{j-1}-X_{j}\right|_{1}^{\alpha} \geq s_{p}^{-\alpha} R^{\alpha+2}\right) .
$$

With the help of (4.4), the rest of the proof is similar to the above.

Thanks to the condition (i), every path satisfying $H_{R}^{\eta}(X)=0$ has probability at most

$$
c_{1}^{R} \exp \left\{-c_{2} s_{p}^{-\alpha}\left(\mu_{1}-\epsilon\right) R\right\}
$$


under $P\left(\cdot \mid X_{0}=y\right)$. On the other hand, condition (ii) ensures that there are at most $\left(4^{d+1} R^{d M}\right)^{R}$ such paths which, in addition, stay inside $[0, R] \times s_{p}^{-1}\left[-2 R^{M}, 2 R^{M}\right]$. Therefore we have

$$
P_{y}\left(H_{R}^{\eta}=0, \max _{1 \leq l \leq R}\left|X_{l}\right|<2 s_{p}^{-1} R^{M}\right) \leq\left(c_{1} 4^{d+1} R^{d M}\right)^{R} \exp \left\{-c_{2} s_{p}^{-\alpha}\left(\mu_{1}-\epsilon\right) R\right\}
$$

and since $s_{p}$ tends to zero as $p \uparrow 1$, the assertion follows.

Let $\psi_{\epsilon}(k, x)=c_{2}\left(\mu_{1}-2 \epsilon\right) 1_{\{(k R, x) \text { is } \epsilon \text {-good }\}}$ and

$$
\Gamma=\left\{\gamma=\left(j, \gamma_{j}\right)_{j \in \mathbb{Z}_{+}}: \gamma_{0}=0, \gamma_{j} \in 2 \mathbb{Z}^{d}\right\}
$$

For $\gamma \in \Gamma$ and an integer $v \geq 1$, we define

$$
J_{v}(\gamma)=\sum_{j=0}^{v-1} \max \left\{\psi_{\epsilon}\left(j, \gamma_{j}\right), C_{5} R\left(\left|\gamma_{j}-\gamma_{j+1}\right|_{\infty}-1\right)_{+}^{\alpha}\right\}
$$

Lemma 7. Let $R$ and $p$ be as in Lemma 5 and 6 . Then for any $v \geq 1$ and $\gamma \in \Gamma$,

$$
\begin{gathered}
P\left(H_{v R}^{\eta}=0 \text { and } X_{j R} \in C_{p}\left(\gamma_{j}\right) \text { for } j=1, \ldots, v\right) \\
\leq \exp \left\{-s_{p}^{-\alpha} J_{v}(\gamma) R\right\} .
\end{gathered}
$$

Proof. We use Markov property at times $R, 2 R, \ldots,(v-1) R$ to bound the left-hand side by

$$
\prod_{j=0}^{v-1} \sup _{y \in C_{p}\left(\gamma_{j}\right)} P_{y}\left(H_{R}^{\theta_{j R} \eta}=0 \text { and } X_{R} \in C_{p}\left(\gamma_{j+1}\right)\right),
$$

where $\theta_{k}(k \in \mathbb{N})$ is the time-shift operator acting on the space of environments. By Lemma 6, it immediately follows that

$$
\sup _{y \in C_{p}\left(\gamma_{j}\right)} P_{y}\left(H_{R}^{\theta_{j R} \eta}=0\right) \leq \exp \left\{-s_{p}^{-\alpha} \psi_{\epsilon}\left(j, \gamma_{j}\right) R\right\}
$$

for sufficiently large $R$. On the other hand, one can show

$$
\sup _{y \in C_{p}\left(\gamma_{j}\right)} P_{y}\left(X_{R} \in C_{p}\left(\gamma_{j+1}\right)\right) \leq \exp \left\{-C_{5} s_{p}^{-\alpha} R^{2}\left(\left|\gamma_{j}-\gamma_{j+1}\right|_{\infty}-1\right)_{+}^{\alpha}\right\}
$$

for large $R$ in the same way as that for (4.3).

This lemma gives a control only for a fixed $\gamma$ but we can indeed reduce the problem to a single $\gamma$ as follows: We have for any $\epsilon \in(0,1)$ that

$$
J_{v}(\gamma) \geq(1-\epsilon) J_{v}(\gamma)+\epsilon C_{5} R \sum_{j=0}^{v-1}\left(\left|\gamma_{j}-\gamma_{j+1}\right|_{\infty}-1\right)_{+}^{\alpha} .
$$


When $p$ is so close to 1 that $s_{p}^{-\alpha} \epsilon C_{5} R^{2} \geq 1$, for some $c>0$ depending only on $d$ and $\alpha$,

$$
\begin{aligned}
\sum_{\gamma \in \Gamma} \exp \left\{-s_{p}^{-\alpha} \epsilon C_{5} R^{2} \sum_{j=0}^{v-1}\left(\left|\gamma_{j}-\gamma_{j+1}\right|_{\infty}-1\right)_{+}^{\alpha}\right\} & \leq \sum_{\gamma \in \Gamma} \exp \left\{-\sum_{j=0}^{v-1}\left(\left|\gamma_{j}-\gamma_{j+1}\right|_{\infty}-1\right)_{+}^{\alpha}\right\} \\
& \leq \exp \{c v\} .
\end{aligned}
$$

Thus it follows that

$$
\sum_{\gamma \in \Gamma} \exp \left\{-s_{p}^{-\alpha} J_{v}(\gamma) R\right\} \leq \exp \left\{-(1-\epsilon) s_{p}^{-\alpha} \inf _{\gamma \in \Gamma} J_{v}(\gamma) R+c v\right\} .
$$

To conclude the proof of the upper bound, it remains to show

$$
\liminf _{v \rightarrow \infty} \frac{1}{v} \inf _{\gamma \in \Gamma} J_{v}(\gamma) \geq c_{2}\left(\mu_{1}-2 \epsilon\right)(1-\epsilon)
$$

almost surely. Without the infimum over $\gamma$, the above is a consequence of the law of large numbers together with Lemma 5 . We indeed have the tail bound

$$
\begin{aligned}
& Q\left(J_{v}(\gamma)<c_{2}\left(\mu_{1}-2 \epsilon\right)(1-\epsilon) v\right) \\
& \quad \leq Q\left(\sum_{j=0}^{v-1} 1_{\left\{\left(j, \gamma_{j}\right) \text { is } \epsilon \text {-good }\right\}}<(1-\epsilon) v\right) \\
& \quad \leq\left(\frac{Q((0,0) \text { is not } \epsilon \text {-good })}{\epsilon}\right)^{\epsilon v}\left(\frac{1}{1-\epsilon}\right)^{(1-\epsilon) v-1}
\end{aligned}
$$

by Bernstein's inequality. The right-hand side is $o(\exp \{-c v\})$ for any $c>0$ when $R$ is sufficiently large, due to Lemma 5 . We show that the infimum has no effect by counting the number of relevant $\gamma$ 's. Obviously we can restrict our consideration to those $\gamma$ with

$$
\sum_{j=0}^{v-1}\left(\left|\gamma_{j}-\gamma_{j+1}\right|_{\infty}-1\right)_{+}^{\alpha} \leq 2\left(\mu_{1}-2 \epsilon\right)(1-\epsilon) v /\left(C_{5} R\right) .
$$

Since we can find $c \geq 1$ such that $x^{\alpha} \leq c(x-1)_{+}^{\alpha}+c$ for $x \geq 0$, the above implies

$$
\sum_{j=0}^{v-1} d^{-\alpha}\left|\gamma_{j}-\gamma_{j+1}\right|_{1}^{\alpha} \leq 2 c v
$$

for all sufficiently large $R>0$. We bound the number of such sequences by

$$
\begin{aligned}
& \#\left\{\left(\gamma_{0}=0, \gamma_{1}, \ldots, \gamma_{v}\right): \sum_{j=0}^{v-1} d^{-\alpha}\left|\gamma_{j}-\gamma_{j+1}\right|_{1}^{\alpha} \leq 2 c v\right\} \\
& \leq \#\left\{\left(\gamma_{0}=0, \gamma_{1}, \ldots, \gamma_{v}\right): \sum_{j=0}^{v-1} \sum_{i=1}^{d}\left|\gamma_{j}^{(i)}-\gamma_{j+1}^{(i)}\right|^{\alpha} \leq c^{\prime} v\right\},
\end{aligned}
$$


where $\gamma_{j}^{(i)}$ stands for $i$-th coordinate of $\gamma_{j}$. Indeed, when $\alpha \leq 1$ this holds with $c^{\prime}=2 c d$ as a consequence of the concavity of $x \mapsto x^{\alpha}$ and, when $\alpha>1$ with $c^{\prime}=2 c d^{\alpha}$ by $\sum_{1 \leq i \leq d}\left|x_{i}\right|^{\alpha} \leq\left(\sum_{1 \leq i \leq d}\left|x_{i}\right|\right)^{\alpha}$. The right-hand side of (4.6) is nothing but the volume of

$$
\bigcup_{x \in \mathbb{Z}^{d v}:|x|_{\alpha}^{\alpha} \leq c^{\prime} v} x+[0,1]^{d v},
$$

where $|x|_{\alpha}=\left(\sum_{i=1}^{d v}\left|x_{i}\right|^{\alpha}\right)^{1 / \alpha}$. As any point $y$ in $x+[0,1]^{d v}$ satisfies

$$
|y|_{\alpha}^{\alpha} \leq \sum_{j=1}^{d v} 2^{\alpha}\left(\left|x_{j}\right|^{\alpha}+1\right) \leq 2^{\alpha+2} c^{\prime} v
$$

the right-hand side of (4.6) is bounded by the volume of $l^{\alpha}$-ball in $\mathbb{R}^{d v}$ with radius $\left(2^{\alpha+2} c^{\prime} v\right)^{1 / \alpha}$, which is known to be

$$
\frac{\left(2\left(2^{\alpha+2} c^{\prime} v\right)^{1 / \alpha} \Gamma(1+1 / \alpha)\right)^{d v}}{\Gamma(1+d v / \alpha)} .
$$

One can check by using Stirling's formula that this is only exponentially large in $v$. Therefore, with the help of (4.5), we find that

$$
Q\left(\inf _{\gamma \in \Gamma} J_{v}(\gamma)<c_{2}\left(\mu_{1}-2 \epsilon\right)(1-\epsilon) v\right)
$$

decays exponentially in $v$ when $R$ is sufficiently large.

Remark 4. We explain how to modify the above block argument to prove $\mu_{p}>0$ for $p \in(0,1]$. We first replace the condition (i) of the $\epsilon$-good box $(\epsilon \in(0,1))$ by

$$
\Phi_{R}\left(\omega_{p}-\left(k, R^{M} x\right)\right) \geq \epsilon
$$

and drop (ii). With this modified definition of $\epsilon$-good box, it is simple to check that the following variant of Lemma 5 holds for general $p \in(0,1]$ :

$$
\lim _{\epsilon \downarrow 0} \limsup _{R \rightarrow \infty} Q((k, x) \text { is } \epsilon \text {-good })=1 .
$$

Next we replace $J_{v}(\gamma)$ for $\gamma \in \Gamma$ by

$$
J_{v}^{\prime}(\gamma)=\sum_{j=0}^{v-1} \max \left\{\epsilon 1_{\{(k, x) \text { is } \epsilon \text {-good }\}}, C_{5}^{\prime} R\left(\left|\gamma_{j}-\gamma_{j+1}\right|_{\infty}-1\right)_{+}^{\alpha}\right\} .
$$

If $C_{5}^{\prime}$ is sufficiently small, we can easily verify that any minimizing path $\pi_{n}$ for $T_{n}\left(\omega_{p}\right)$ with $\pi_{n}(j R) \in C_{p}\left(\gamma_{j}\right)(0 \leq j \leq n / R)$ has passage time larger than $J_{v}^{\prime}(\gamma)$. Therefore we get

$$
T_{n}\left(\omega_{p}\right) \geq \inf _{\gamma \in \Gamma} J_{[n / R]-1}^{\prime}(\gamma)
$$

and, when $R \in \mathbb{N}$ is chosen sufficiently large and $\epsilon$ small, we have

$$
\liminf _{v \rightarrow \infty} \frac{1}{v} \inf _{\gamma \in \Gamma} J_{v}^{\prime}(\gamma)>0
$$


in exactly the same way as above.

\section{APPENDIX}

We provide a proof of (1.3) for completeness. We consider $d=1$ case first since the other case will reduce to it. Set

$$
\mathcal{L}=\{(m, x) \in \mathbb{N} \times \mathbb{Z}: m+x \in 2 \mathbb{Z}\} .
$$

For $R>0$ and $(m, x) \in \mathcal{L}$, we say $(m, x)$ is open if there exists a $y_{m} \in R x+$ $(-R, R) \cap \mathbb{Z}$ such that $\eta\left(m, y_{m}\right)=0$. It is easy to see that

$$
Q((m, x) \text { is open }) \rightarrow 1
$$

as $R \rightarrow \infty$. Thus when $R$ is large, the directed site percolation on $\mathcal{L}$ is supercritical and we can find a percolation point $(1, x) \in \mathcal{L}$. This implies that there exists a path $\left\{\left(k, y_{k}\right)\right\}_{k \geq 1}$ satisfying

$$
\eta\left(k, y_{k}\right)=0 \text { and }\left|y_{k+1}-y_{k+2}\right| \leq 3 R
$$

for all $k \geq 1$. Then it follows that

$$
\begin{aligned}
\liminf _{n \rightarrow \infty} \frac{1}{n} \log Z_{n}^{\eta,-\infty} & \geq \liminf _{n \rightarrow \infty} \frac{1}{n} \log P\left(X_{k}=y_{k} \text { for all } k \leq n\right) \\
& \geq-c_{2} 3^{\alpha} R^{\alpha} .
\end{aligned}
$$

For the case $d \geq 2$, we have

$$
Z_{n}^{\eta,-\infty} \geq P\left(H_{n}^{\eta}=0 \text { and } X_{k} \in \mathbb{Z} \times\{0\}^{d-1} \text { for all } 1 \leq k \leq n\right)
$$

and the right-hand side can be bounded from below in the same way as for $d=1$.

\section{ACKNOWLEDGEMENTS}

The first author was partially supported by CNRS, UMR 7599. The second author was supported by JSPS KAKENHI Grant Number 24740055. The fourth author was supported by JSPS KAKENHI Grant Number 25400136.

\section{REFERENCES}

[1] Amir, G., Corwin, I., Quastel, J.: Probability distribution of the free energy of the continuum directed random polymer in 1+1 dimensions. Comm. Pure Appl. Math. 64(4), 466-537 (2011)

[2] Boucheron, S., Lugosi, G., Massart, P.: Concentration inequalities. Oxford University Press, Oxford (2013).

[3] Carmona, P., Hu, Y.: On the partition function of a directed polymer in a Gaussian random environment. Probab. Theory Related Fields 124(3), 431-457 (2002)

[4] Carmona, R., Koralov, L., Molchanov, S.: Asymptotics for the almost sure Lyapunov exponent for the solution of the parabolic Anderson problem. Random Oper. Stochastic Equations 9(1), 77-86 (2001)

[5] Carmona, R.A., Molchanov, S.A.: Parabolic Anderson problem and intermittency. Mem. Amer. Math. Soc. 108(518), viii+125 (1994)

[6] Comets, F., Cranston, M.: Overlaps and pathwise localization in the Anderson polymer model. Stochastic Process. Appl. 123(6), 2446-2471 (2013). 
[7] Comets, F., Popov, S., Vachkovskaia, M.: The number of open paths in an oriented $\rho$ percolation model. J. Stat. Phys. 131(2), 357-379 (2008)

[8] Comets, F., Shiga, T., Yoshida, N.: Directed polymers in a random environment: path localization and strong disorder. Bernoulli 9(4), 705-723 (2003)

[9] Comets, F., Yoshida, N.: Brownian directed polymers in random environment. Comm. Math. Phys. 254(2), 257-287 (2005)

[10] Comets, F., Yoshida, N.: Directed polymers in random environment are diffusive at weak disorder. Ann. Probab. 34(5), 1746-1770 (2006)

[11] Comets, F., Yoshida, N.: Localization transition for polymers in Poissonian medium. Comm. Math. Phys. 323(1), 417-447 (2013).

[12] Cox, J.T.: The time constant of first-passage percolation on the square lattice. Adv. in Appl. Probab. 12(4), 864-879 (1980)

[13] Cox, J.T., Kesten, H.: On the continuity of the time constant of first-passage percolation. J. Appl. Probab. 18(4), 809-819 (1981)

[14] Cranston, M., Mountford, T.S.: Lyapunov exponent for the parabolic Anderson model in $\mathbf{R}^{d}$. J. Funct. Anal. 236(1), 78-119 (2006).

[15] Cranston, M., Mountford, T.S., Shiga, T.: Lyapunov exponents for the parabolic Anderson model. Acta Math. Univ. Comenian. (N.S.) 71(2), 163-188 (2002)

[16] Cranston, M., Mountford, T.S., Shiga, T.: Lyapunov exponent for the parabolic Anderson model with Lévy noise. Probab. Theory Related Fields 132(3), 321-355 (2005).

[17] Darling, R.W.R.: The Lyapunov exponent for products of infinite-dimensional random matrices. In: Lyapunov exponents (Oberwolfach, 1990), Lecture Notes in Math., vol. 1486, pp. 206-215. Springer, Berlin (1991).

[18] Fukushima, R., Yoshida, N.: On exponential growth for a certain class of linear systems. ALEA Lat. Am. J. Probab. Math. Stat. 9(2), 323-336 (2012)

[19] Garet, O., Gouéré, J.B., Marchand, R.: The number of open paths in oriented percolation (2015). preprint, arXiv:1312.2571

[20] Griffeath, D.: The binary contact path process. Ann. Probab. 11(3), 692-705 (1983)

[21] Howard, C.D., Newman, C.M.: Euclidean models of first-passage percolation. Probab. Theory Related Fields 108(2), 153-170 (1997)

[22] Howard, C.D., Newman, C.M.: Geodesics and spanning trees for Euclidean first-passage percolation. Ann. Probab. 29(2), 577-623 (2001)

[23] Kasahara, Y.: Tauberian theorems of exponential type. J. Math. Kyoto Univ. 18(2), 209-219 (1978)

[24] Kesten, H.: Aspects of first passage percolation. In: École d'été de probabilités de Saint-Flour, XIV-1984, Lecture Notes in Math., vol. 1180, pp. 125-264. Springer, Berlin (1986)

[25] Kesten, H., Sidoravicius, V.: A problem in last-passage percolation. Braz. J. Probab. Stat. 24(2), 300-320 (2010)

[26] Lacoin, H.: Existence of an intermediate phase for oriented percolation. Electron. J. Probab. 17, no. 41, 17 (2012)

[27] Moriarty, J., O'Connell, N.: On the free energy of a directed polymer in a Brownian environment. Markov Process. Related Fields 13(2), 251-266 (2007)

[28] Mountford, T.S.: A note on limiting behaviour of disastrous environment exponents. Electron. J. Probab. 6, no. 1, 9 pp. (electronic) (2001)

[29] Nagaev, S.V.: Large deviations of sums of independent random variables. Ann. Probab. 7(5), 745-789 (1979)

[30] Seppäläinen, T.: Scaling for a one-dimensional directed polymer with boundary conditions. Ann. Probab. 40(1), 19-73 (2012). 
24 FRANCIS COMETS, RYOKI FUKUSHIMA, SHUTA NAKAJIMA, AND NOBUO YOSHIDA

[31] Shiga, T.: Exponential decay rate of survival probability in a disastrous random environment. Probab. Theory Related Fields 108(3), 417-439 (1997).

[32] Yoshida, N.: Phase transitions for the growth rate of linear stochastic evolutions. J. Stat. Phys. 133(6), 1033-1058 (2008)

(Francis Comets) Laboratoire Probabilités et Modélisation Aléatoire, Université PARis Diderot - PARis 7, PARis, France

E-mail address: comets@math.univ-paris-diderot.fr

(Ryoki Fukushima) Research Institute in Mathematical Sciences, Kyoto University, KYOTO, JAPAN

E-mail address: ryoki@kurims.kyoto-u.ac.jp

(Shuta Nakajima) Research Institute in Mathematical Sciences, Kyoto University, KYOTO, JAPAN

E-mail address: njima@kurims.kyoto-u.ac.jp

(Nobuo Yoshida) Graduate School of Mathematics, Nagoya University, Nagoya, JAPAN

E-mail address: noby@math.nagoya-u.ac.jp 